Veranlassung sich nach außen verschiebe, und zwar befinde sich beim Beugen die Kniescheibe in der richtigen Lage, während beim Strecken dieselbe ganz nach außen ginge.

Bei der Untersuchung fand sich nun Folgendes: beim Beugen und Strecken der Beine gleiten die beiden Kniescheiben hin und her, und zwar stehen in Beugestellung dieselben fast in ihrer normalen Lage, während sie beim Strecken nach außen lnxieren. Wird in Streckstellung die Kniescheibe in ihre richtige Lage gebracht und so festgehalten so ist ein Heben des Unterschenkels unmöglich. Läßt man die Kniescheibe los und spannt Patient die Quadricepssehne an so luxiert die Patella sofort wieder nach außen, und so ist Patient im stande, das Bein in gestreckter Stellung 7.u heben. Es besteht starke Atrophie beider Oberschenkel. Beide Kniegelenke sind vollkommen frei beweg. lich, jedoch fühlt man bei Bewegungen ein deutliches Knacken in den Gelenken. Es besteht große Schwäche besonders im linken Bein. Die seitlichen Kniegelenksbänder sind gelockert. Bewegungen in den Ge-

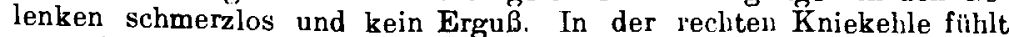
man einen derben Strang, und bei genauer Palpation lassen sich mehrere verschieden große, harte Körper hin- und herschieben. Die ubrigen freien Körper siud bei der Untersuchung gerade nicht zu fühlen. Zur genaueren Feststellung der etwa in den Gelenken befindlichen Fremdkörper werden Röntgenbilder aufgenommen, die die Größe und Jage der vorhandenen Gelenkkörper dentlich erkennen lassen:

Figur I. Rechtes Knie in Streckstellung von vorn.

\section{Ein Fall von freien Gelenkkörpern in beiden Kniegelenken mit doppelseitiger habitueller Luxation der Patella nach außen.)}

Von Dr. W. Böcker, Spezialarzt für orthopadische Chirurgie in Berlin.

M. H.! Ich wollte mir erlauben, kurz über einen seltenen, interessanten Fall von knorpeligen, knöchernen Gelenkkörpern in beiden Kniegelenken mit doppelseitiger habitueller Patellarluxation nach außen, der kïrzlich in der Klinik meines verehrten Chefs, des Herrn Geheimlat Prof. Dr. Hoffa zur Operation kam, zu berichten. Dabei werde ich die Geschichte der Gelenkkörper übergehen, da sie ja in einer ganzen Reihe interessanter Arbeiten hinlänglich Erwähnung findet. Auch werde ich nicht, da die histologischen Untersuchungen der freien Gelenkkörper noch nicht abgeschlossen sind, im einzelnen auf die Genese derselben eingehen. Die Frage der Genese ist schon viel besprochen und besonders, seit König im Jahre 1887 unter dem Namen der Osteochondritis dissecans aufs neue ein Krankheitsbild beschrieben hat und einen großen Teil der bis jetzt als traumatisch aufgefaßten Fälle von freien Gelenkkörpern durch einen eigentümlichen, von ihm selbst nicht zu erklärenden dissezierenden Prozeß entstanden wissen wollte, Gegenstand lebhafter Diskussion unter den Chirurgen geworden, aber bis heute noch nicht erledigt. So findet man Anhänger und Gegner dieser Königschen Anschauung über die Osteochondritis dissecans in der Literatur vertreten. Es kann daher nur wünschenswert sein, solche Fälle, die geeignet sind, als Beweismaterial für oder gegen eine Ansicht zu dienen, zu veröfentlichen und dadurch zur Klärung der verschiedenen Meinungen beizutragen.

Onser Fall scheint nun auf Grund der erhobenen Anamnese und des klinischen Befundes für die Königsche Theorie zu sprechen und interessiert einmal, weil die Gelenkkörper in beiden Kniegelenken sich finden und ein Teil derselben in die Bursa gastrocnemio-semimembranosa rechts gewandert ist, zweitens weil gleichzeitig eine doppelseitige habituelle Luxation der Patella besteht und drittens wegen des Ergebnisses der in einer Sitzung an beiden Kniegelenken ausgeführten Operation. Zunächst möchte ich kurz auf die Kranken. geschichte eingehen.

Vor etwa 20 Jahren, also im Jahre 1883, stellten sich bei dem Patienten im Alter von 13 Jahren ohne ein bekanntes Trauma plötzlich Schmerzen im linken Kniegelenk ein, die ihn hinderten, das Bein zu bewegen. Diese Beschwerden kehrten in Zwischenräumen immer wieder und boten das typische Bild der Einklemmung. Einige Jahre später wurde auch von ihm selbst ein beweglicher Körper an der Außenseite der Kniescheibe gefühlt. Im Jahre 1886 traten nun auch die gleichen Beschwerden im rechten Kniegelenk auf, wofür er keine Ursache anzugeben weiß. Einige Jahre später etwa, im Jahre 1893, fühlte er in der rechten Kniekehle eine Verdickung, die ihm aber keine weiteren Beschwerden machte. Auch in diesem Gelenk will er kurze Zeit darauf einen Körper an der Außenseite der Kniescheibe hin- und hergeschoben haben. Mit diesen beweglichen Körpern im Gelenk ist Patient jahrelang herumgelaufen, teils mit den heftigsten Einklemmungserscheinungen, teils auch ohne Beschwerden. Die Kniee sind häufig angeschwollen gewesen. In dieser Zeit will nun Patient auch bemerkt haben, daß seine Kniescheibe an beiden Seiten ohne eine besondere Berlins.

1) Vortrag, gehalten am 14. Dezember 1903 in der Freien Vereinigung der Chirurgen
Fig. 1.

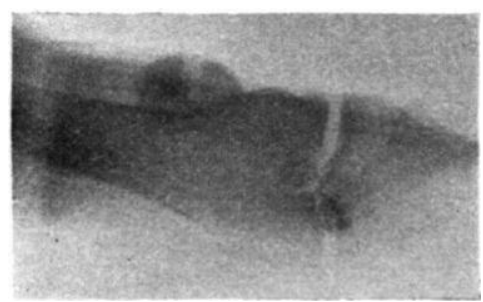

Auf demselben sieht man die Patella dentlich nach außen luxiert und in der Fossa intercondylica einen rundlichen, etwa kirschengroßen Schatten; außerdem sind mehrere kleinere, gleichfalls rundliche Schatten von Haselnuß- bis Hïhnerschrotgröße nach innen zu im Gelenkspalt gelegen.

Figur 1I. Linkes Knie in Streckstellung von vorn. Iinks sieht man die Patella ebenfalls nach außen luxiert und in der Fossa inter

Fig. 2.

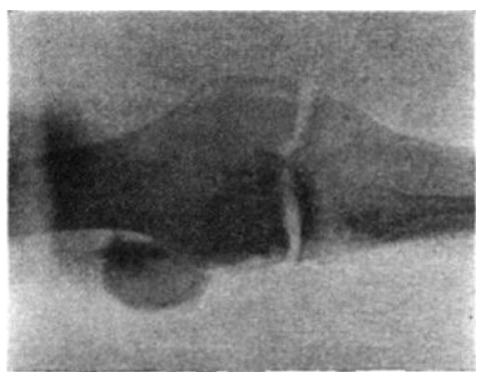

condylica einen haselnußgroßen und am inneren Condylus am Kniegelenksspalt einen kleinwalnußgroßen Schatten.

Figur III Das linke Knie in Beugestellung von innen. Auf dem-

Fig. 3 .

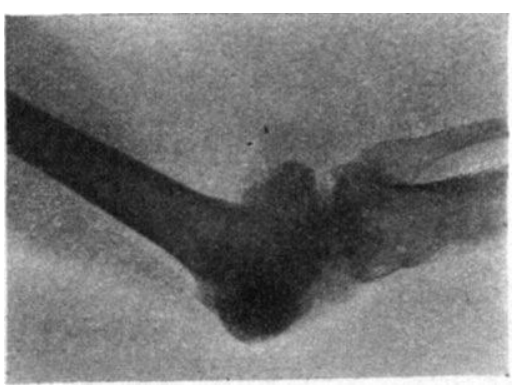

selben sieht man im Gelenkspalt nach vorn einen haselnußgroßen Schatten und einen zweiten etwas größeren am inneren Condylus.

Bei der am 9. November vorgenommenen Operation zeigt sich nun ein den Röntgenbildern entsprechender Befund: wir haben die Operation beschlossen, einmal um die Gelenkkörper zu entfernen und zweitens, um die habituelle Luxation zu beseitigen. Wir sind hierbei zur Beseitigung der Luxation von dem Gedanken ausgegangen, den 
Semimembranosus loszulösen and die Sehne desselben mit dem iuneren oberen erschlafften Rand der Patella zu vernähen, um so für den Quadriceps ein Gegengewicht herzustellen. Beide Kniee werden in einer Sitzung operiert. Zuerst wird in der rechten Kniekehle nach

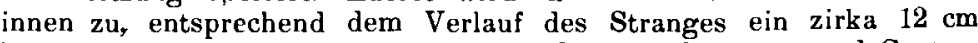
langer Schnitt gemacht und zwischen Semimembranosus und Gastrocnemius eingegangen, wobei der zwischen diesen Muskeln gelegene, häufig mit dem Gelenk kommunizierende Schleimbeutel freigelegt wird. Bei Eröffnung desselben fließt etwas Synovialflüssigkeit aus, und kommen einige Gelenkkörper zum Vorschein. Daraif werden die übrigen im Schleimbeutel befindlichen Corpora mobilia mit dem scharfen Löffel herausgeholt, und der Schleimbeutel selbst wird exstirpiert. Es waren im ganzen acht Gelenkkörper, von himbeerartiger Form, die hühnerschrot- bis haselnußgrols waren und ein glänzendes, knorpeliges Aussehen hatten. Darauf wurde der Semimembranosus losgelöst, an seiner Insertionsstelle abgetrennt und auf die Patella übergepflanzt zwecks Fixierung der nach außen luxierten Patella. Hierauf folgt die Eröffnung des Gelenks an der Innenseite der Patella durch einen zirka $13 \mathrm{~cm}$ langen Schnitt, wobei man am Condylus internus zwischen dem inneren Rand der Trochlea und dem Epicondylus deutlich einen unregelmäßigen, zirka $4 \mathrm{~cm}$ im Durchmesser großen Defekt sieht, dessen Grund bereits mit Knorpel ausgekleidet war, dessen Rand insbesondere am Rand der Trochlea rauh, scharf und gezackt sich anfuhlt. Dieser hervorstehende Rand wird mit dem Meißiel entfernt und so geglättet. Er besteht aus Knorpel und Knochen, wie im Röntgenbild zu sehen ist. Der Defekt entsprach in Form und Größe dem bereits gelösten Gelenkkörper, der im oberen äußeren Abschnitt des Recessus aufgefunden wurde. Der Gelenkkörper war zirka $4 \mathrm{~cm}$ lang, $31 / . \mathrm{cm}$ breit und $1 \mathrm{~cm}$ dick, unregelmäßig, höckerig, von glänzendem Aussehen und knorpeliger Beschaffenheit. Ich möchte ihn dem A ussehen nach mit einem Seestern vergleichen. Im übrigen konnten Veränderungen im Gelenk nicht nachgewiesen werden. Auch war sonst nichts zu finden, woher die im Schleimbeutel befindlichen Körper stammten. Wir nehmen an, daß dieselben sich von dem Defekt los. gelöst haben, weil sie das gleiche Aussehen zeigen und in den Schleimbeutel eingewandert sind. Darauf wurde die Sehne des Semimembranosus vom Gelenkschnitt aus mit einer Kornzange subkutan nach vorn gezogen und mit dem inneren oberen Rand der reponierten Patella vernäht. In der gleichen Weise wird auch links verfahren. Nach Eröffnung des Gelenks findet sich gleichfalls am inneren Condylus an korrespondierender Stelle zwischen dem inneren Rand der Trochlea und dem Epicondylus ein etwa $5 \mathrm{~cm}$ im Durchmesser großer, unregulmäßiger, auf dem Grund mit Knorpel ausgekleideter Defekt mit rauhen und gezackten Rändern, der etwa in Form und Größe einem bereits gelösten, im oberen äuberen Recessus liegenden Gelenkkörper von 5 cın Länge, $4 \mathrm{~cm}$ Breite und $1 \% \mathrm{~cm}$ Dicke entsprach. Ich möchte diesen Gelenkkörper mit einer Koralle vergleichen. Derselbe ist ganz unregelmäßig, höckerig, von glänzendem Aussehen und knorpeliger Beschaffenheit. Ein zweiter Gelenkkörper von zirka Kleinwalnußßgröße fand sich vorn am Kniegelenksspalt in der Fossa intercondylica und hilı noch durch einen dünnen Stiel mit Synovia und Fett zusammen. Derselbe wurde mit dem Stiel und einem Stück Fett herausgenommen. Er war glatt und von rundlicher Fornı, dabei an beiden Seiten etwas abgeschliffen durch die Reibungen an der Patella und den Condylen. Dieser Körper machte den Eindruck eines Knochens, was auch durch das Röntgenbild bestätigt wurde, das ganz dentlich Knochenst rukturen zeigte, während sämtliche uibrigen Körper auch auf der Röntgenplatte sich als Knorpel erwiesen. Bezüglich der Herkunft dieses Körpers dürfen wir wohl annehmen, daß er sich ebenfalls von dem vorhandenen Defekt langsam losgelöst hat, da sich im Gelenk sonst keine Ursprungsstelle finden laBit, und an einer anderen Stelle des Gelenks mit der Synovia verwachsen ist. Wissen wir doch, da $B$ derartige Verwachsungen nicht selten vorkommen. Irgend welche Zeichen von Arthritis deformans waren nicht vorhanden

Was die habituelle Luxation der Patellae anlangt, deren es drei gibt, die traumatische, kongenitale und patlıologische, so können wir die ersten beiden von vornherein ausschließjen, da sicher kein Trauma vorliegt und die Luxation sich erst mit 23 Jahren, wie Patient be stimmt weil\}, ausgebildet hat. Das Zustandekommen der pathologischen Luxation, die hier in Frage kommt, wird nach H of fa zurück. geführt einmal auf eine Veränderung in der Konfiguration der Gelenkenden und zweitens auf eine Erweiterung, respektive Erschlaffung der Kniegelenkskapsel. In unserem Falle führe ich das Zustandekommen derselben auf eine solche Erweiterung und Schlaffheit der $K$ apsel infolge des häufig durch die Gelenkkörper aufgetretenen Keizes in selben und dadurch entstandenen Hydrops und auf die infolge der Atrophie des Quadrizeps erschlaffte Sehne desselben zurück. Ich nıöchte somit die Luxation mit dem Gelenkleiden in Zusammenhang briıgen, respektive als eine Folge desselben ansehen.

Wenn wir nun das Resumee ziehen, so dürfen wir wohl annehmen, wenn auch das Martenssche Symptom des allmähliclien Beginnens der Beschwerden im affizierten Gelenk hier weg fällt und das Ergebnis der histologischen Untersuchung noch aussteht, dab es sich um eine spontane Osteochondritis dissecans handelt, und laBt sich dieser
Fall für die von anderer Seite angefochtene Theorie Königs verwerten. So vertritt in erster Linie Barth auf Grund seiner histologischen und experimentellen Untersuchungen die Ansicht, daß Gelenkkörper nur auf traumatischem Wege oder durch Arthritis deformans entstehen. Seinen Ausführungen schlieBt sich auch Follbrecht an. In der jüngst erschienenen Arbeit von Börner aus der Rostocker Klinik wird die Entstehungsursache von freien Gelenkkörpern auf Grund seiner Untersuchungen auf irgend ein Trauma zurückgeführt. wenn auch ein solches in der Anamnese mit Bestinmtheit fehlt, da bestimmte Anhaltspunkte fïr entzündliche Prozesse in seinen sämitlichen Fällen mikroskopisch sich nicht fintlen lassen. Dagegen hält Schuchardt die Entstehung clıondraler Gelenkmäuse unabhängig von Verletzungen für sicher und sagt, dal 3 solche Gelenkkörper nur durch einen krankhaften Prozeł am Knochen erklärt werden könuen. Nur die von König in Aussicht gestellten Resultate seiner patloologisclianatomischen Forschungen werden wohl bald mehr Licht ïber die vielumstrittene Frage verbreiten.

Daß ich mich in unserem Falle der Anschauung Königs zuneige, dafür spricht 1. das Fehlen eines Traumas in der Allamnese, worauf eben König im Gegensatz zu Anderen und mit ilını Marteus einen besonderen Wert legen, gestützt auf das reichlaaltige Material, 2. der Sitz und die GröBe des Prozesses, 3. die Tatsache, daß an korrespondierender. Stelle am inneren rechten und linken Condylus femoris die Loslösung der Gelenkkörper erfolgt ist - denn es wäre doch zum mindesten auffallend, ganz abgesehen dawon, daß mit Bestimmtheit ein Trauma in Abrede gestellt wird, wenn ein Trauma an zwei gleichen Stellen der beiden inneren Kondy้len eine Loslösung der Gelenkkörper verursacht hätte, dann aber hätte anch meiner Ansicht nach nur eine schwere Gewalteinwirkung, wie sie König fordert, einen solchen Defekt, wie ihn unser Fall zeirt, setzen künnen - 4. das ganze Aussehen, die Form und Bescliaffenheit (ler Defekte und gefundenen freien Gelenkkörper. - Interessant ist noch, diul3 ein Teil der Knorpel in den Schleimbentel gewandert ist, und dïrfte dies imınerhin zu den Seltenheiten gelıören. Mir ist nur ein Fall von $\mathbf{B l a n k e}$ bekannt, wo dieselben in die Bursa subcruralis gewandert sind. Das Leiden beschränkt siclı nach Martens fast aussclılielBlich auf ein Gelenk. Derselbe erwähnt nur einen Fall, wo beide Ellenbogengelenke einen abgelösten Celenkkörper enthalten haben. Ueber das Sehicksal der Patienten spricht er sich günstig aus

Soneit mir die Literatur zur Verfügung stınd, habe ich einen solchen Fall, wie den unsrigen, noch nicht heschirieben gefuncleı, und verdient dieser Fall darum, besonders beachtet zu werden.

Wenu ich nun zun Schluß3 noch einige Worte iiber die Operation und das Resultat derselben hinzufüge, so soll man sich liente nicht mehr scheuen, beide Gelenke in einer Sitzung zu operieren, mul zwa dieselben, wenn erforderlich, in weiter Ausdelınung zu eröffnell, da so der Patient nit einem Male von seinem Leiden befreit ist unul das Innere des Gelenks einer grïndliclien Besichtigung unterzogen werdeıl kann. Denn nur so kann mau sich nach Möglichkeit ein klares Bild von der Beschaffenheit des Gelenks verscliaffen und Spuren aufdecken, die sonst dem Auge verborgen bleiben. Die geringe Gefalır der Ankylose, insbesondere das befriedigencle Resultat, wie unser Fall in der kurzen Zeit es beweist, soll dazu aufmuntern. Stets soll man aber der Worte Königs eingedenk sein, "fingerlos zu operieren“. Was nun die Fixation der Patella durch Vernähung mit dem abgelösten Semimembranosus zwecks Beseitigung der habituellen Luxation betrifft, so duirfte diese Methode, wie aus der Literatur hervorgeht, noch nicht geübt sein, und möclite ich diese Plastik infolge giinstigeri-Resul tates in geeigneten Fällen zur Nachprüfung aufs wärnste empfehlen.

Wie Sie sich. meine Herren, selbst überzeugen kïnnen, kann der Patient bereits wieder fiinf Wochen nach der Operation ohne $\mathrm{Be}$ schwerden herumgehen und trotz des bedeutenden Fingriffs die Kniee bis zu einem halben Rechten beugen. Die beirlen Kniescheiben sirıd gut fixiert und dabei in normalen Grenzen verschieblich. Beide Beine können jetzt bei normaler Lage der Kniescheibe grestreckt gelobeu werden. Bei weiterer zweckentsprechender Behandlung mit Massage und Gymnastik verspricht das Resultat ein vollkommen norinales und gutes zu werden. 